Research Paper

\title{
Surrogate endpoints shortening the therapeutic evaluation duration for different subgroups of patients with nasopharyngeal carcinoma receiving intensity-modulated radiotherapy: A retrospective analysis of 830 patients stratified by the 8th edition of the UICC/AJCC staging system and plasma Epstein-Barr viral
}

Shu Zhou ${ }^{1,2^{*}}$, Chen Chen ${ }^{1,2^{*}}$, Song-ran Liu ${ }^{1,2}$, Ya-lan Tao ${ }^{1,2}$, Hui Chang ${ }^{1,2}$, Xiao-hui Wang ${ }^{1,2}$, Xin Yang ${ }^{1,2}$, Wen-wen Zhang ${ }^{1,2}$, Shan Liu 1,2, Shi-rong Ding1,2, Guan-nan Wang1,2, and Yun-fei Xia1,2®

1. State Key Laboratory of Oncology in South China, Collaborative Innovation Center for Cancer Medicine, Sun Yat-sen University Cancer Center, Guangzhou 510060, Guangdong, P. R. China.

2. Department of Radiation Oncology, Sun Yat-sen University Cancer Center, Guangzhou 510060, Guangdong, P. R. China.

*These authors contributed equally to this work.

$\triangle$ Corresponding author: Yun-fei Xia, PhD, MD, State Key Laboratory of Oncology in South China; Department of Radiation Oncology, Sun Yat-sen University Cancer Center, 651 Dongfeng Road East, Guangzhou 510060, P. R. China. Tel.: +86-20-87343373, Fax: +86-20-87343373, Email: xiayf1962@126.com

(c) Ivyspring International Publisher. This is an open access article distributed under the terms of the Creative Commons Attribution (CC BY-NC) license (https://creativecommons.org/licenses/by-nc/4.0/). See http://ivyspring.com/terms for full terms and conditions.

Received: 2018.02.13; Accepted: 2018.07.17; Published: 2018.09.08

\begin{abstract}
Purpose: Investigating surrogate endpoints shortening the time of therapeutic evaluation in nasopharyngeal carcinoma (NPC) after radical treatment.

Patients and Methods: We retrospectively analyzed 830 patients receiving intensity-modulated radiotherapy (IMRT) from 2008 to 2010 and being stratified by the 8th edition of UICC/AJCC staging system and the plasma Epstein-Barr virus DNA (EBV DNA). The annual rates of overall survival (OS), progression-free survival (PFS), loco-regional recurrence-free survival (LRFS), and distant metastasis-free survival (DMFS) were sequentially calculated using the life table and compared by the McNemar method.

Results: The time of shortening therapeutic evaluation by surrogate endpoints: OS, PFS, LRFS and DMFS could be shortened to 1 -year $(100 \%$ vs $100 \%, P=1)$ in patients with stage $\mathrm{I}$; OS, PFS, LRFS and DMFS could be shortened to 3 -year $(96.9 \%$ vs $96.1 \%, P=1 ; 94.6 \%$ vs $92.2 \%, P=0.125 ; 96.9 \%$ vs $95.3 \%, P=0.5)$ and $4-y e a r(92.2 \%$ vs $91.2 \%, P=0.125)$ in stage II; In the high EBV DNA group, OS and DMFS could be shortened to 1 -year $(100 \%$ vs $100 \%, P=1 ; 100 \%$ vs $100 \%, P=0.25)$ in stage Il; OS and PFS could be shortened to 3 -year $(94.3 \%$ vs $91.4 \%, P=1 ; 82.9 \%$ vs $74.3 \%, P=0.25)$ in stage III; OS could be shortened to 4 -year $(75 \%$ vs $72.7 \%, P=1)$ in stage IVA.

Conclusions: The time of therapeutic evaluation could be shortened to $<5$-year in stages I-II patients. The year of surrogate endpoints could be ahead in stages II-IVA with high EBV DNA.

Key words: nasopharyngeal carcinoma; surrogate endpoints; intensity-modulated radiotherapy; the 8th edition of UICC/AJCC staging; Epstein-Barr Viral DNA; overall survival; progression-free survival; loco-regional recurrence-free survival; distant metastasis-free survival
\end{abstract}




\section{Introduction}

Nasopharyngeal carcinoma (NPC) is a malignant tumor originating from nasopharyngeal epithelium, with a unique geographical distribution pattern and high incidence in Southeast Asia and southern China, especially in Guangdong [1-4]. Over the past 30 years, the overall survival rate of nasopharyngeal carcinoma has been significantly improved due to the advances in radiotherapy and the extensive use of chemotherapy $[5,6]$. Presently, the 5-year survival rate of nasopharyngeal carcinoma is approximately $80-90 \%$, with a local control rate of $90 \%$ or more. The distant metastasis free survival rate was $66 \%-84 \%$ [7].Locoregional recurrence and distant metastasis at the time of 5 years after treatment accounted for nearly $100 \%$ [8]. Therefore, 5-year survival rate is used as the foremost endpoint in clinical trials $[9,10]$.

The 5-year survival endpoint was objective, accurate and convenient to analyze and was the most reliable clinical trial endpoint. However, there are several disadvantages, including requirements for a large sample size, high medical cost and prolonging the clinical trial proposal-validation-period. At the same time, non-tumor-related death during follow-up, as well as some of the rescue treatment after the progression, will have a bias on the endpoint. In the era of individualized treatment, utilizing the 5 -year survival endpoint has many limitations, and more and more oncologists are beginning to study surrogate endpoints instead of the "true clinical outcome" [11-14].

Previously, a study about surrogate endpoints shortening the time of therapeutic evaluation with nasopharyngeal carcinoma conducted by our group provided evidence supporting the use of overall survival (OS) and loco-regional control (LRC) endpoints $<5$ years as surrogate endpoints [15]. The research by the Meta-Analysis of Chemotherapy in Nasopharynx Carcinoma (MAC-NPC) Collaborative Group demonstrated that two-year PFS and distant metastasis-free survival (DMFS) are valid surrogate end points for five-year OS to assess the treatment effects of chemotherapy in loco-regionally advanced NPC [16].Nonetheless, none of the previous studies, including our previous study, have mentioned plasma Epstein-Barr virus DNA (EBV DNA). At present, pretreatment EBV load has been confirmed to be greatly associated with patient outcome and can be used as a strong prognostic biomarker [17-19].

Recently, the 8th edition of TNM staging system was published to further help clinicians evaluate the prognosis and assign proper treatment [20,21]. Tang LL et al [22] indicated a better segregation of survival curves in NPC patients using the 8th edition system compared with those using the 7th edition system, which interpreted these results as a strong indicator to apply the new edition in clinical practice and trials.

Based on the new staging system and the importance of EBV DNA, the aim of the present study is to investigate surrogate endpoints to shorten the time of therapeutic evaluation for different subgroup of patients with NPC receiving IMRT.

\section{Materials and Methods}

\section{Patients}

We continuously reviewed the medical records of 3894 patients who were diagnosed with NPC in the Sun Yat-Sen University Cancer Center (Guangzhou, China) during the period between January 2008 and December 2010. The inclusion criteria for this study were: (1) Newly diagnosed M0 stage patients. (2) Receiving intensity modulated radiation therapy (IMRT). (3) Epstein-Barr virus related test data was complete. (4) No lost follow-up within 5 years. According to the above criteria, our study excluded 455 patients with non-M0 stage nasopharyngeal carcinoma, 2080 patients who did not receive IMRT, 481 patients without complete Epstein-Barr virus test data and 48 patients lost follow-up within 5 years. A total of 830 patients met the criteria and were enrolled in this retrospective clinical study. All patients were restaged according to the 8th edition of the UICC/AJCC staging system and the patient data was collected by two physicians specializing in nasopharyngeal cancer. Any divergences were resolved by consensus.

\section{Treatment}

Patients enrolled in this study underwent radical IMRT. Prescribed radiation dose was 2.0 to 2.27 Gy per fraction with 5 daily fractions per week for 6 to 7 weeks. Cumulative doses were 66 Gy or greater to the primary tumor and $\geq 50$ Gy to the bilateral cervical lymph nodes and potential sites of local infiltration.

Chemotherapy included induction, concomitant and adjuvant chemotherapy. The choice of chemotherapy regimen and the number of courses used were determined by the clinicians. The chemotherapy regimen was mainly platinum based.

\section{Follow-up and Endpoints}

The patients were followed up with by phone and/or in the outpatient clinic. The follow-up items included survival status, local failure and distant metastasis. All the events were confirmed by pathological examination and/or imaging. The last date of follow-up was May 2017. Four common used survival endpoints were selected, overall survival (OS), progression-free survival (PFS), loco-regional recurrence-free survival (LRFS), and distant 
metastasis-free survival (DMFS). OS was defined as time from diagnosis to death from any cause. PFS was defined as time from diagnosis to loco-regional failure, distant failure, or death from any cause, whichever occurred first. LRFS was defined as time from diagnosis to loco-regional failure. DMFS was defined as time from diagnosis to distant failure.

\section{Study design}

The flowchart of our study design is presented in Fig 1. All patients were divided into three groups according to levels of EBV DNA measured: low EBV DNA $\left(<10^{3}\right.$ copies $\left./ \mathrm{ml}\right)$, mid EBV DNA $\left(\geq 10^{3}\right.$ copies $/ \mathrm{ml}$ and $<10^{5}$ copies/ml), high EBV DNA ( $\geq 10^{5}$ copies $/ \mathrm{ml}$ ), plus all the patients as a group, for a total of four groups. We determined $10^{3}$ copies $/ \mathrm{ml}$ and $10^{5}$ copies/ml as the cut-off values of EBV DNA, which are similar to those measured in previous studies [23]. The patients in the four groups were then stratified by the 8th edition TNM staging system. We calculated and compared the survival rates of the different subgroups at 4 and 5 years. If a subgroup exhibited no significant differences between the 4- and 5-year survival rates, we further calculated the 3-, 2- and 1-year survival rates and compared these to the 5-year survival rate. The survival rate of the earliest years with the indifferent comparison is reduced by 5-year survival rate. The earliest year survival rate could be selected as surrogate endpoints to the 5-year survival rate if the difference between the two rates was less than one standard deviation (the standard deviation is calculated from the 5 different year survival rates).

\section{Statistical Analysis}

The survival rates were calculated by life table. Survival curves were drawn using the Kaplan-Meier method with the two-sided log-rank test. Survival rate comparisons were performed with the McNemar's test. All the tests were two-tailed and a P-value $<0.05$ was considered to indicate a statistically significant difference. The statistical analyses were performed with Statistical Product and Service Solutions software version 22.0 (SPSS Inc., Chicago, IL, USA).

\section{Results}

The baseline characteristics of the patients are summarized in Table 1 . In the EBV DNA $\geq 10^{5}$ group, there was only one patient at stage $\mathrm{T} 1$, three patients at stage N0, and no patients at stage I. The annual survival rate and the $\mathrm{P}$ value between years and the earliest year of surrogate endpoint are presented in Table 2-4. The survival curve by TNM clinical staging is presented in Fig 2-5.

\section{Overall Survival}

For stage I patients, the 1-year OS rate could be selected as the surrogate endpoint to the 5-year OS rate $(100 \%$ vs $100 \%, P=1)$. For stage II patients, the 3 -year OS rate could be selected as the surrogate endpoint to the 5 -year OS rate $(96.9 \%$ vs $96.1 \%, \mathrm{P}=1)$.

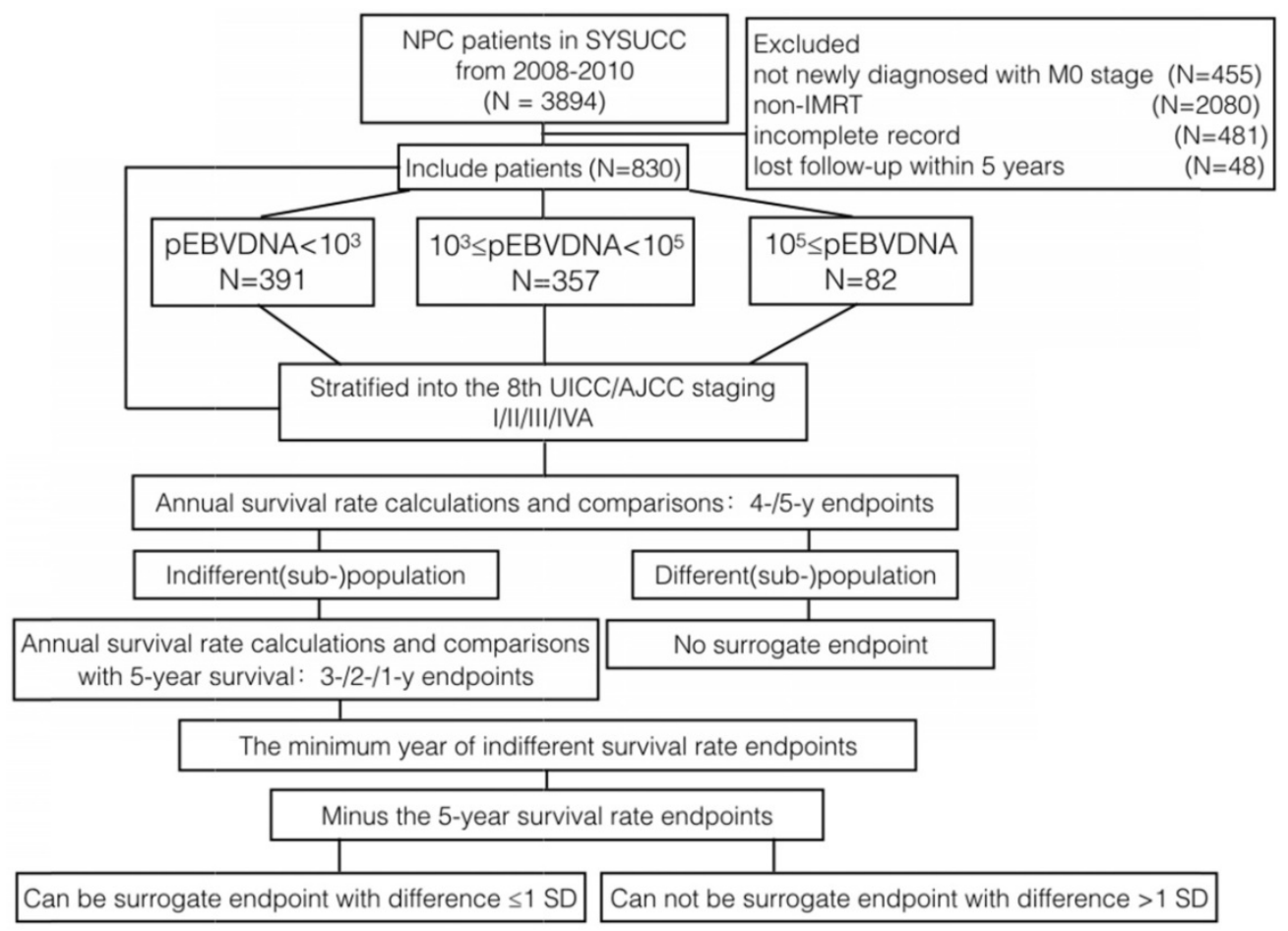

Fig 1. Flowchart of the study design. NPC, nasopharyngeal carcinoma; EBV DNA, pretreatment plasma Epstein-Barr viral deoxyribonucleic acid; IMRT, intensity-modulated; OS, overall survival; PFS, progression-free survival; LRFS, loco-regional recurrence-free survival; DMFS, distant metastasis-free survival; McNemar: analysis of variance; SD, standard deviation 
For patients with any other stage, no annual OS rate less than the 5-year survival rate could be selected as the surrogate endpoint.

Table 1. Baseline Patient Demographic and Clinical Characteristics

\begin{tabular}{lllll}
\hline & Total patients & EBV $<103$ & $103 \leq \mathrm{EBV}<10^{5}$ & $\mathrm{EBV} \geq 10^{5}$ \\
\hline & $\mathrm{n}=830$ & $\mathrm{n}=391(47.1 \%)$ & $\mathrm{n}=357(43 \%)$ & $\mathrm{n}=82(9.9 \%)$ \\
\hline $\begin{array}{l}\text { Age (years) } \\
\text { median }\end{array}$ & $45(13-78)$ & $44(13-76)$ & $45(13-77)$ & $45(24-78)$ \\
male & $590(71.1 \%)$ & $283(72.4 \%)$ & $251(70.3 \%)$ & $56(68.3 \%)$ \\
female & $240(28.9 \%)$ & $108(27.6 \%)$ & $106(29.7 \%)$ & $26(31.7 \%)$ \\
Tumor stage & & & & \\
T1 & $60(7.2 \%)$ & $42(10.7 \%)$ & $17(4.8 \%)$ & $1(1.2 \%)$ \\
T2 & $187(22.5 \%)$ & $116(29.7 \%)$ & $58(16.2 \%)$ & $13(15.9 \%)$ \\
T3 & $382(46 \%)$ & $166(42.5 \%)$ & $173(48.5 \%)$ & $43(52.4 \%)$ \\
T4 & $201(24.3 \%)$ & $67(17.1 \%)$ & $109(30.5 \%)$ & $25(30.5 \%)$ \\
Node stage & & & & \\
N0 & $100(12 \%)$ & $76(19.4 \%)$ & $21(5.9 \%)$ & $3(3.7 \%)$ \\
N1 & $343(41.3 \%)$ & $189(48.3 \%)$ & $134(37.5 \%)$ & $20(24.4 \%)$ \\
N2 & $320(38.6 \%)$ & $112(28.6 \%)$ & $171(47.9 \%)$ & $37(45.1 \%)$ \\
N3 & $67(8.1)$ & $14(3.6 \%)$ & $31(8.7 \%)$ & $22(26.8 \%)$ \\
TNM stage & & & & \\
I & $18(2.2 \%)$ & $17(4.3 \%)$ & $1(0.3 \%)$ & $0(0 \%)$ \\
II & $129(15.5 \%)$ & $103(26.3 \%)$ & $23(6.4 \%)$ & $3(3.7 \%)$ \\
III & $423(51 \%)$ & $192(49.1 \%)$ & $196(54.9 \%)$ & $35(42.7 \%)$ \\
IVA & $260(31.3 \%)$ & $79(20.3 \%)$ & $137(38.4 \%)$ & $44(53.6 \%)$ \\
FU (months) & & & & \\
median & $84(4-112)$ & & & \\
\hline & & & & \\
\hline
\end{tabular}

Our data indicated that differences in EBV DNA levels did not change the earliest year of surrogate endpoints for stage I patients. However, for stage II patients in the high EBV DNA group, the 1-year OS rate could be selected as the surrogate endpoint to the 5 -year OS rate $(100 \%$ vs $100 \%, P=1)$. For stage III patients in the high EBV DNA group, the 3-year OS rate could be selected as the surrogate endpoint to the 5 -year OS rate $(94.3 \%$ vs $91.4 \%, P=1)$. For stage IVA patients in the high EBV DNA group, the 4-year OS rate could be selected as the surrogate endpoint to the 5 -year OS rate $(75 \%$ vs $72.7 \%, \mathrm{P}=1)$.

\section{Progression-free Survival}

For stage I patients, the 1-year PFS rate could be selected as the surrogate endpoint to the 5-year PFS ( $100 \%$ vs $100 \%, P=1)$. For stage II patients, the 3 -year PFS rate could be selected as the surrogate endpoint to the 5 -year PFS rate $(94.6 \%$ vs $92.2 \%, \mathrm{P}=0.25)$. For patients with any other stage, no annual PFS rate $<5$-year could be selected as the surrogate endpoints.

Our data indicated that the difference in EBV DNA levels did not change the earliest year of surrogate endpoints for patients with stages I, II and IVA. However, for stage III patients in the high EBV DNA group, the 3-year PFS rate could be selected as the surrogate endpoint to the 5-year PFS rate $(82.9 \%$ vs $74.3 \%, \mathrm{P}=0.25)$.

\section{Loco-regional Recurrence-free Survival}

For stage I patients, the 1-year LRFS rate could be selected as the surrogate endpoint to the 5-year LRFS rate $(100 \%$ vs $100 \%, P=1)$. For stage II patients, the 3-year LRFS rate could be selected as the surrogate endpoint to the 5-year LRFS rate $(96.9 \%$ vs $95.3 \%$, $\mathrm{P}=0.5$ ). For stage III patients, the 4-year LRFS rate could be selected as the surrogate endpoint to the 5 -year LRFS rate $(94.7 \%$ vs $93.7 \%, \mathrm{P}=0.125)$. For stage IVA patients, the 3-year LRFS rate could be selected as the surrogate endpoint to the 5-year LRFS rate $(92.3 \%$ vs $90.9 \%, \mathrm{P}=0.25$ ).

Table 2. Annual survival rates and their comparisons of OS and PFS in different subgroups of patients

\begin{tabular}{|c|c|c|c|c|c|c|c|c|c|c|c|c|}
\hline & \multicolumn{6}{|c|}{ OS\% (P-value) } & \multicolumn{6}{|c|}{ PFS\% (P-value) } \\
\hline & 1 year & 2 years & 3 years & 4 years & 5 years & SD & 1 year & 2 years & 3 years & 4 years & 5 years & SD \\
\hline \multicolumn{13}{|c|}{ Total patients } \\
\hline I & $100.0(1.000)$ & $100.0(1.000)$ & $100.0(1.000)$ & $100.0(1.000)$ & 100.0 & 0.0 & $100.0(1.000)$ & $100.0(1.000)$ & $100.0(1.000)$ & $100.0(1.000)$ & 100.0 & 0.0 \\
\hline II & $99.2(0.125)$ & $98.4(0.250)$ & $96.9(1.000)$ & $96.1(1.000)$ & 96.1 & 1.3 & $99.2(0.004)$ & $96.1(0.125)$ & $94.6(0.250)$ & $93.0(1.000)$ & 92.2 & 2.5 \\
\hline III & 99.3 & 97.9 & 96.0 & $94.3(0.004)$ & 92.2 & 2.5 & 97.2 & 92.9 & 89.8 & $87.0(0.008)$ & 85.1 & 4.3 \\
\hline IVA & 96.9 & 91.9 & 87.3 & $79.2(0.004)$ & 75.4 & 7.9 & 91.2 & 82.3 & 75.4 & $71.5(0.031)$ & 69.2 & 8.0 \\
\hline \multicolumn{13}{|c|}{$\mathrm{EBV}<10^{3}$} \\
\hline I & $100.0(1.000)$ & $100.0(1.000)$ & $100.0(1.000)$ & $100.0(1.000)$ & 100.0 & 0.0 & $100.0(1.000)$ & $100.0(1.000)$ & 100.0(1.000) & 100.0(1.000) & 100.0 & 0.0 \\
\hline II & $100.0(0.375)$ & $99.0(0.500)$ & $98.1(1.000)$ & $97.1(1.000)$ & 97.1 & 1.1 & $100.0(0.070)$ & $98.1(0.250)$ & $96.1(0.500)$ & $95.1(1.000)$ & 94.2 & 2.1 \\
\hline III & 99.5 & $97.9(0.016)$ & $96.4(0.063)$ & $95.8(0.125)$ & 93.7 & 2.0 & 97.9 & 94.8 & $91.7(0.031)$ & $90.1(0.25)$ & 88.5 & 3.4 \\
\hline IVA & 100.0 & 97.5 & $94.9(<0.001)$ & $87.3(0.063)$ & 79.7 & 7.4 & 97.5 & 88.6 & $81.0(0.008)$ & $74.7(0.25)$ & 70.9 & 9.6 \\
\hline \multicolumn{13}{|c|}{$10^{3} \leq \mathrm{EBV}<10^{5}$} \\
\hline I & $100.0(1.000)$ & $100.0(1.000)$ & $100.0(1.000)$ & $100.0(1.000)$ & 100.0 & 0.0 & $100.0(1.000)$ & $100.0(1.000)$ & $100.0(1.000)$ & $100.0(1.000)$ & 100.0 & 0.0 \\
\hline II & $95.7(1.000)$ & $95.7(1.000)$ & $91.3(1.000)$ & $91.3(1.000)$ & 91.3 & 2.2 & $95.7(0.500)$ & $87.0(1.000)$ & $87.0(1.000)$ & $87.0(1.000)$ & 87.0 & 3.5 \\
\hline III & 99.0 & 97.4 & $95.9(<0.001)$ & $93.4(0.063)$ & 90.8 & 2.9 & 95.9 & 92.3 & $89.3(<0.001)$ & $85.7(0.125)$ & 83.7 & 4.4 \\
\hline IVA & 95.6 & 90.5 & $84.7(<0.001)$ & $75.9(0.250)$ & 73.7 & 8.4 & 89.1 & $80.3(<0.001)$ & $73.7(0.125)$ & $71.5(1.000)$ & 70.8 & 6.9 \\
\hline \multicolumn{13}{|c|}{$E B V \geq 10^{5}$} \\
\hline \multicolumn{13}{|c|}{ I } \\
\hline II & $100.0(1.000)$ & $100.0(1.000)$ & 100.0(1.000) & $100.0(1.000)$ & 100.0 & 0.0 & $100.0(1.000)$ & $100.0(1.000)$ & $100.0(1.000)$ & 66.7(1.000) & 66.7 & 16.3 \\
\hline III & $100.0(0.375)$ & $100.0(0.375)$ & $94.3(1.000)$ & $91.4(1.000)$ & 91.4 & 3.9 & $100.0(0.012)$ & $85.7(0.125)$ & $82.9(0.250)$ & 77.1(1.000) & 74.3 & 9.0 \\
\hline IVA & $95.5(0.002)$ & $86.4(0.063)$ & $81.8(0.125)$ & $75.0(1.000)$ & 72.7 & 8.2 & 86.4 & $77.3(0.031)$ & $70.5(0.250)$ & $65.9(0.500)$ & 61.4 & 8.8 \\
\hline
\end{tabular}

EBV: Epstein-Barr viral; OS: overall survival; PFS: progression-free survival; SD: standard deviation is calculated from five annual survival rates; P-value: Comparisons between survival rates until the difference is statistically significant 
Table 3. Annual survival rates and their comparisons of LRFS and DMFS in different subgroups of patients

\begin{tabular}{|c|c|c|c|c|c|c|c|c|c|c|c|c|}
\hline & \multicolumn{6}{|c|}{ LRFS\% (P-value) } & \multicolumn{6}{|c|}{ DMFS\% (P-value) } \\
\hline & 1 year & 2 years & 3 years & 4 years & 5 years & SD & 1 year & 2 years & 3 years & 4 years & 5 years & SD \\
\hline \multicolumn{13}{|c|}{ Total patients } \\
\hline I & $100.0(1.000)$ & $100.0(1.000)$ & $100.0(1.000)$ & $100.0(1.000)$ & 100.0 & 0.0 & $100.0(1.000)$ & $100.0(1.000)$ & $100.0(1.000)$ & $100.0(1.000)$ & 100.0 & 0.0 \\
\hline II & $100.0(0.070)$ & $97.6(0.500)$ & $96.9(0.500)$ & $96.1(1.000)$ & 95.3 & 1.6 & $99.2(0.250)$ & $98.4(0.250)$ & $98.4(0.500)$ & $96.9(1.000)$ & 96.1 & 1.1 \\
\hline III & 99.5 & 98.1 & $96.1(0.002)$ & $94.7(0.125)$ & 93.7 & 2.1 & 97.9 & 95.3 & $93.6(0.002)$ & $92.2(0.125)$ & 91.2 & 2.4 \\
\hline IVA & 98.4 & $95.2(0.004)$ & $92.3(0.250)$ & $91.4(1.000)$ & 90.9 & 2.8 & 93.0 & $86.0(<0.001)$ & $82.5(0.125)$ & $80.8(1.000)$ & 80.4 & 4.7 \\
\hline \multicolumn{13}{|c|}{$\mathrm{EBV}<10^{3}$} \\
\hline I & $100.0(1.000)$ & $100.0(1.000)$ & $100.0(1.000)$ & $100.0(1.000)$ & 100.0 & 0.0 & $100.0(1.000)$ & $100.0(1.000)$ & $100.0(1.000)$ & $100.0(1.000)$ & 100.0 & 0.0 \\
\hline II & $100.0(0.375)$ & $99.0(1.000)$ & $98.0(1.000)$ & $98.0(1.000)$ & 97.0 & 1.0 & $100.0(0.219)$ & $99.0(0.250)$ & $99.0(0.250)$ & $97.1(1.000)$ & 96.1 & 1.4 \\
\hline III & 99.5 & $99.5(0.004)$ & $97.3(0.063)$ & $95.7(0.500)$ & 94.6 & 2.0 & $98.4(0.004)$ & $95.8(0.250)$ & $94.8(0.500)$ & $94.3(1.000)$ & 93.7 & 1.7 \\
\hline IVA & $100.0(0.125)$ & $97.5(0.250)$ & $94.9(1.000)$ & $93.5(1.000)$ & 93.5 & 2.5 & 97.5 & $89.9(0.016)$ & $84.8(0.250)$ & $80.9(1.000)$ & 80.9 & 6.3 \\
\hline \multicolumn{13}{|c|}{$10^{3} \leq \mathrm{EBV}<10^{5}$} \\
\hline I & $100.0(1.000)$ & $100.0(1.000)$ & 100.0(1.000) & 100.0(1.000) & 100.0 & 0.0 & $100.0(1.000)$ & $100.0(1.000)$ & $100.0(1.000)$ & $100.0(1.000)$ & 100.0 & 0.0 \\
\hline II & $100.0(0.625)$ & $90.9(1.000)$ & $90.9(1.000)$ & $90.9(1.000)$ & 90.9 & 3.6 & $95.7(1.000)$ & $95.7(1.000)$ & $95.7(1.000)$ & $95.7(1.000)$ & 95.7 & 0.0 \\
\hline III & 99.5 & $98.4(0.008)$ & $96.3(0.125)$ & $95.3(0.500)$ & 94.2 & 2.0 & 96.9 & 94.4 & $92.3(0.031)$ & $90.3(0.500)$ & 89.2 & 2.8 \\
\hline IVA & $97.0(0.008)$ & $94.7(0.125)$ & $91.5(1.000)$ & $91.5(1.000)$ & 90.6 & 2.4 & $92.6(<0.001)$ & $85.2(0.500)$ & $82.9(1.000)$ & $82.9(1.000)$ & 82.9 & 3.8 \\
\hline \multicolumn{13}{|c|}{$E B V \geq 10^{5}$} \\
\hline \multicolumn{13}{|c|}{ I } \\
\hline II & $100.0(1.000)$ & $100.0(1.000)$ & $100.0(1.000)$ & $66.7(1.000)$ & 66.7 & 16.3 & 100.0(1.000) & $100.0(1.000)$ & $100.0(1.000)$ & $100.0(1.000)$ & 100.0 & 0.0 \\
\hline III & $100.0(0.125)$ & $88.6(1.000)$ & $88.6(1.000)$ & $85.6(1.000)$ & 85.6 & 5.3 & $100.0(0.219)$ & $97.1(0.250)$ & $94.2(0.500)$ & $91.3(1.000)$ & 88.4 & 4.1 \\
\hline IVA & $100.0(0.125)$ & $92.6(0.500)$ & $90.0(1.000)$ & $87.3(1.000)$ & 87.3 & 4.7 & $86.4(0.310)$ & $81.8(0.250)$ & 77.2(1.000) & $74.8(1.000)$ & 72.3 & 5.0 \\
\hline
\end{tabular}

Table 4. Year of surrogate endpoints of four groups stratified by TNM staging

\begin{tabular}{|c|c|c|c|c|}
\hline \multirow{2}{*}{$\begin{array}{l}\text { Total patients } \\
\text { 8th staging }\end{array}$} & \multicolumn{4}{|c|}{ Year of surrogate endpoints } \\
\hline & OS & PFS & LRFS & DMFS \\
\hline I & 1 & 1 & 1 & 1 \\
\hline II & 3 & 3 & 3 & 4 \\
\hline III & 5 & 5 & 4 & 4 \\
\hline IVA & 5 & 5 & 3 & 3 \\
\hline \multicolumn{5}{|l|}{$\mathrm{EBV}<10^{3}$} \\
\hline I & 1 & 1 & 1 & 1 \\
\hline II & 3 & 3 & 3 & 4 \\
\hline III & 5 & 4 & 3 & 3 \\
\hline IVA & 5 & 4 & 3 & 3 \\
\hline \multicolumn{5}{|l|}{$10^{3} \leq \mathrm{EBV}<10^{5}$} \\
\hline I & 1 & 1 & 1 & 1 \\
\hline II & 3 & 2 & 2 & 1 \\
\hline III & 4 & 4 & 4 & 4 \\
\hline IVA & 4 & 3 & 3 & 2 \\
\hline \multicolumn{5}{|l|}{$E B V \geq 10^{5}$} \\
\hline \multicolumn{5}{|l|}{ I } \\
\hline II & 1 & 4 & 4 & 1 \\
\hline III & 3 & 3 & 2 & 4 \\
\hline IVA & 4 & 4 & 3 & 3 \\
\hline
\end{tabular}

EBV, plasma Epstein-Barr viral; OS, overall survival; PFS, progression-free survival; LRFS, loco-regional recurrence-free survival; DMFS, distant metastasis-free survival

Our data indicate that the difference in EBV DNA levels did not change the year of surrogate endpoints for patients with stages I-IVA.

\section{Distant Metastasis-free Survival}

For stage I patients, the 1-year DMFS rate could be selected as the surrogate endpoint to the 5-year DMFS rate $(100 \%$ vs $100 \%, P=1)$. For stage II patients, the 4-year DMFS rate could be selected as the surrogate endpoint to the 5-year DMFS rate (96.9\% vs $96.1 \%, P=1)$. For stage III patients, the 4-year DMFS rate could be selected as the surrogate endpoint to the 5 -year DMFS rate $(92.2 \%$ vs $91.2 \%, \mathrm{P}=0.125)$. For stage
IVA patients, the 3-year DMFS rate could be selected as the surrogate endpoint to the 5-year DMFS rate (82.5\% vs $80.4 \%, \mathrm{P}=0.125)$.

Our data indicate that the difference in EBV DNA levels did not change the earliest year of surrogate endpoints for patients with stages I, III-IVA. However, for stage II patients in the high EBV DNA group, the 1-year DMFS rate could be selected as the surrogate endpoint to the 5 -year DMFS rate $(100 \%$ vs $100 \%, P=1)$.

\section{Discussion}

To the best of our knowledge, this is the first study to investigate surrogate endpoints including OS, PFS, LRFS and DMFS for patients with NPC stratified by the 8th edition of the UICC/AJCC staging system and EBV DNA in the IMRT era. Our research demonstrated that OS, LRFS, DMFS and LRFS at <5-year endpoints were mainly equivalent with 5-year endpoints for stage I and II patients. Moreover, high EBV DNA enabled the time of surrogate endpoints to advance for stage II-IVA patients.

A previous study that was undertaken by our group identified surrogate endpoints for stage I, which represent stable and good outcomes; and for stage N3, which represent stable but poor outcomes [15]. However, the majority of the patients in that study were treated by conventional radiotherapy, which made the result applicable only to conventional radiotherapy treatments. Furthermore, EBV DNA, which is closely related to the prognosis, was not taken into account. Additionally, PFS and DMFS were not included in the analysis. 
A

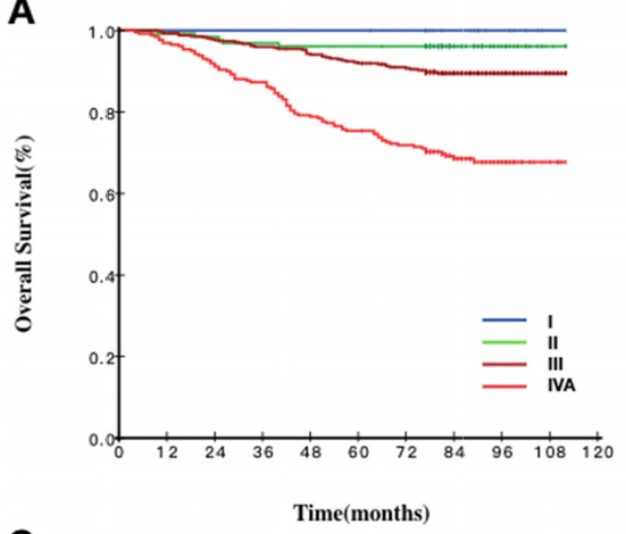

C

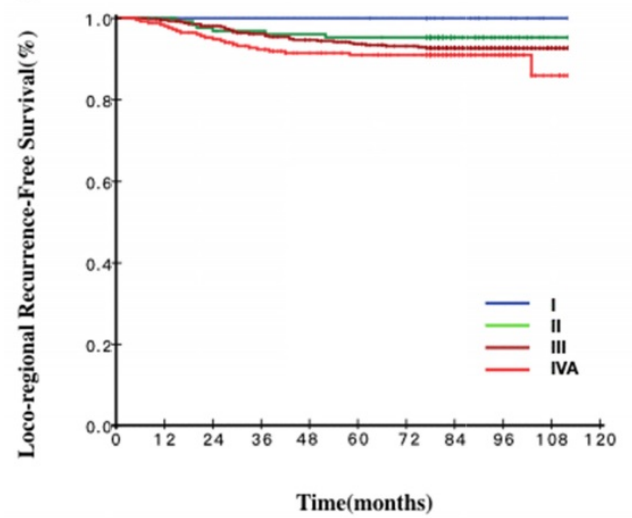

B
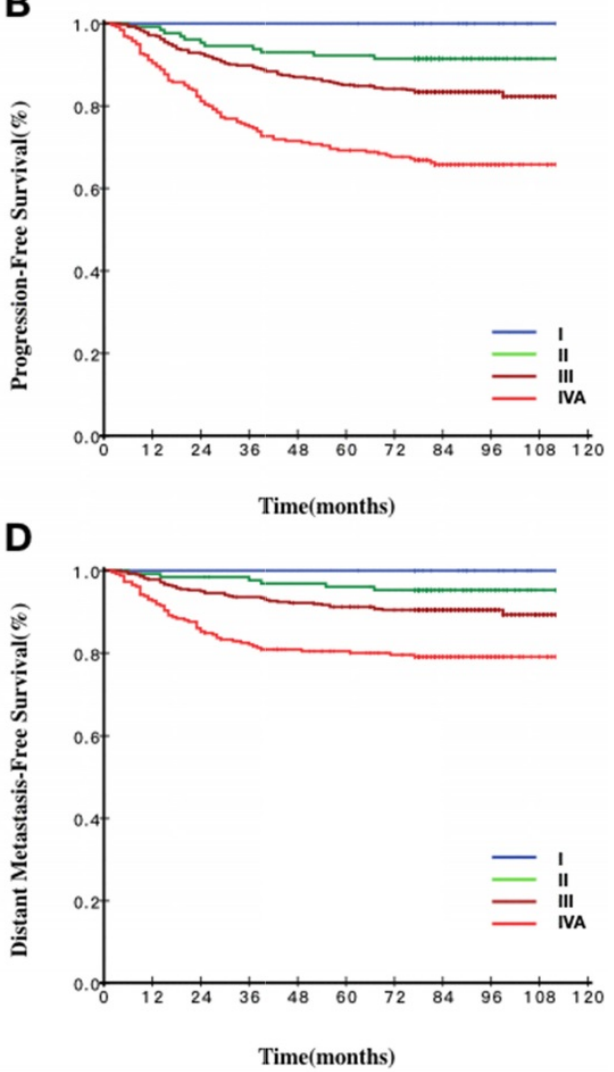

Fig 2. Survival curves stratified by the $8^{\text {th }}$ TNM staging of all patients. A Overall survival curves of patients according to TNM staging. B Progression-free survival curves of patients according to TNM staging. C Loco-regional recurrence-free survival curves of patients according to TNM staging. D Distant metastasis-free survival curves of patients according to TNM staging.

A

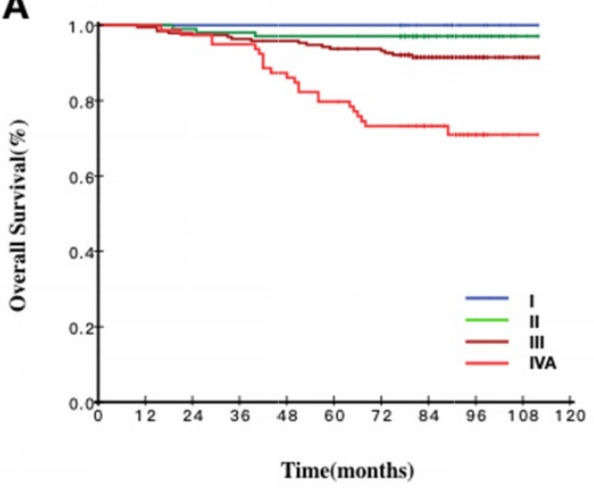

C

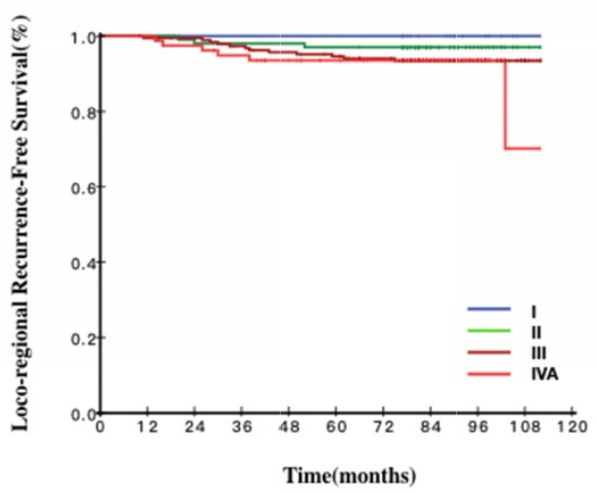

B

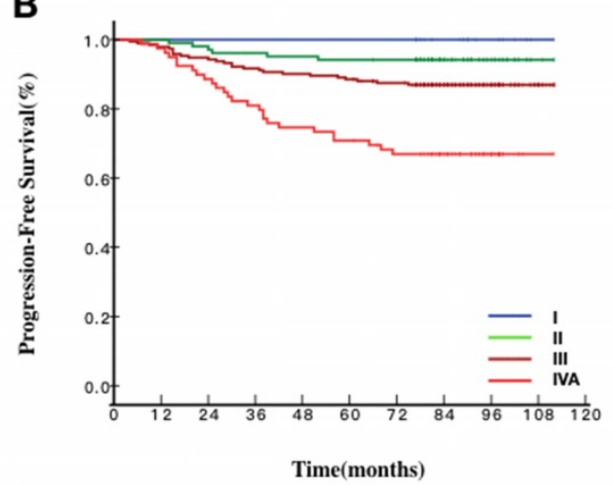

D

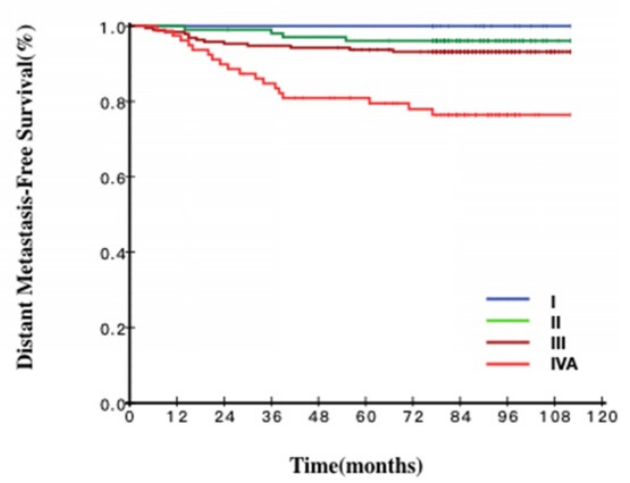

Fig 3. Survival curves stratified by the $8^{\text {th }}$ TNM staging of patients with EBV DNA< $10^{3}$ copies/ml. A Overall survival curves of patients according to TNM staging. B Progression-free survival curves of patients according to TNM staging. C Loco-regional recurrence-free survival curves of patients according to TNM staging. D Distant metastasis-free survival curves of patients according to TNM staging. 

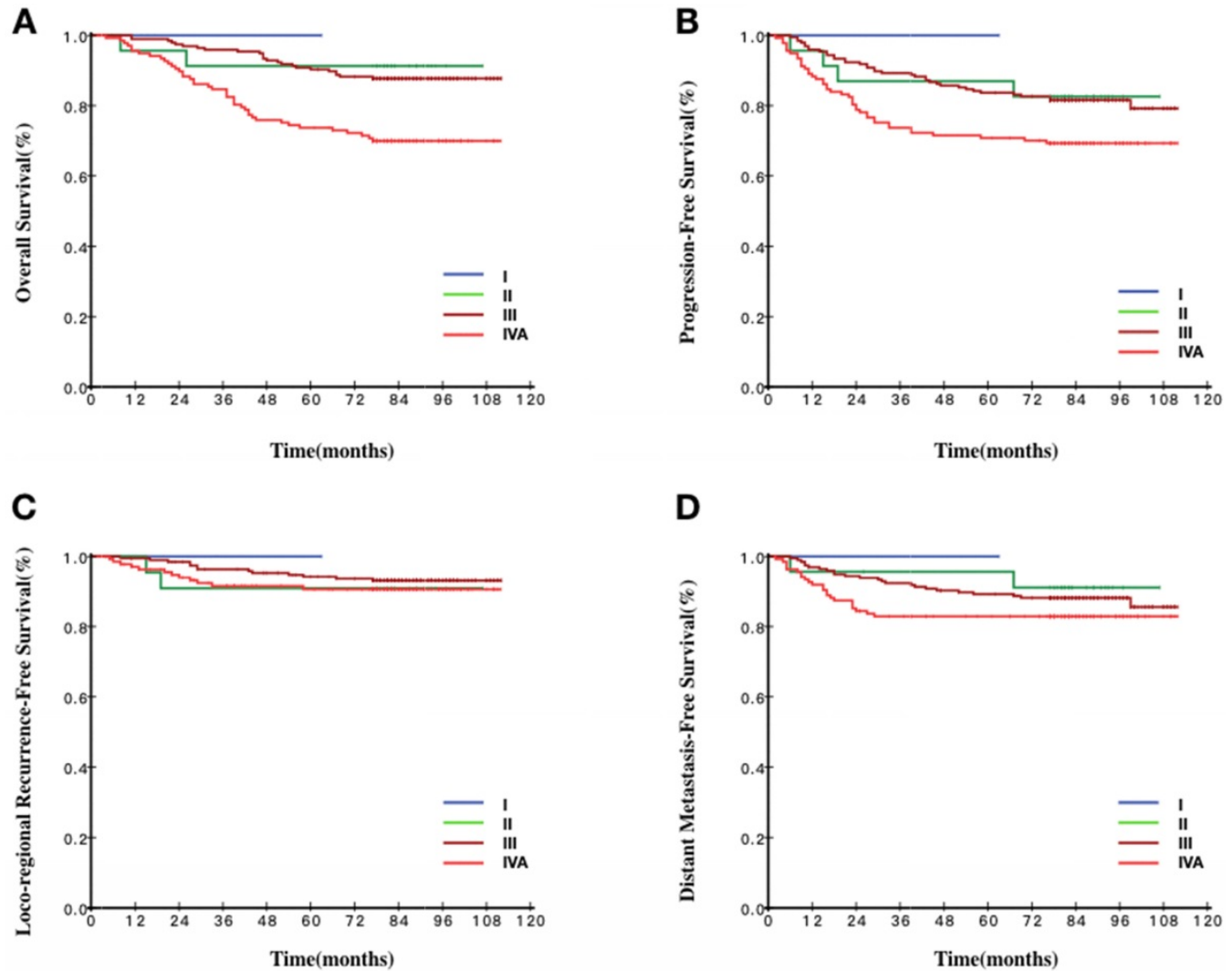

Fig 4. Survival curves stratified by the $8^{\text {th }}$ TNM staging of patients with EBV DNA $\geq 10^{3}$ copies $/ \mathrm{ml}$ and $<10^{5}$ copies/ml. A Overall survival curves of patients according to TNM staging. B Progression-free survival curves of patients according to TNM staging. C Loco-regional recurrence-free survival curves of patients according to TNM staging. D Distant metastasis-free survival curves of patients according to TNM staging.

A

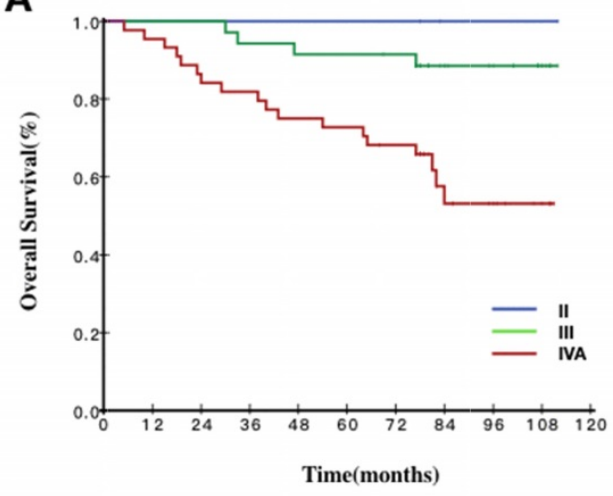

C

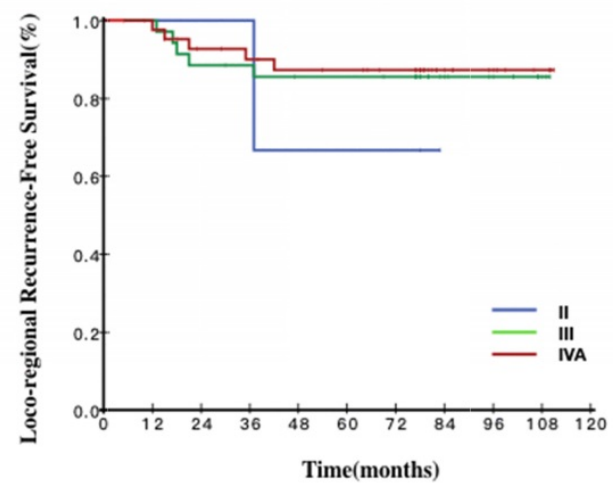

B

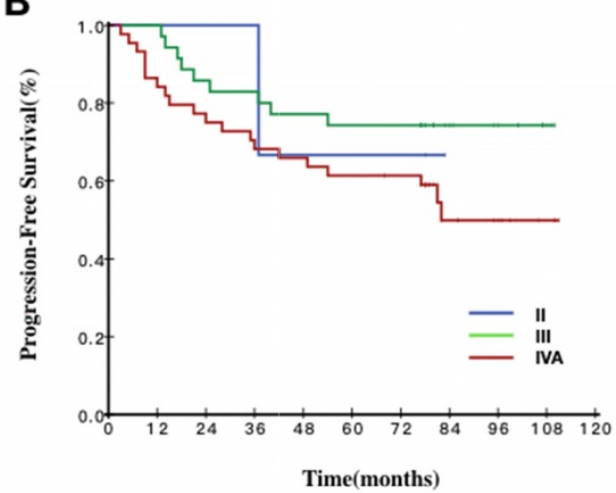

D

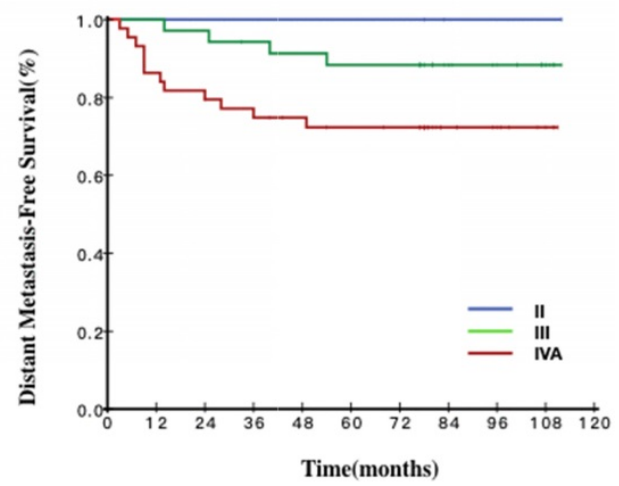

Fig 5. Survival curves stratified by the $8^{\text {th }}$ TNM staging of patients with EBV DNA $\geq 10^{5}$ copies/ml. A Overall survival curves of patients according to TNM staging. B Progression-free survival curves of patients according to TNM staging. C Loco-regional recurrence-free survival curves of patients according to TNM staging. D Distant metastasis-free survival curves of patients according to TNM staging. 
Our data suggested that, for stage I patients, all 1-year endpoints could be used as surrogate endpoints. As shown in Fig 2, the survival curves for stage I were smooth prior to year 5 . The same phenomenon was also observed for the 1- to 5-year survival rates in different EBV DNA groups. A number of previous studies reported excellent outcomes in stage I patients $[15,17,24]$. In our study, the OS, PFS, DMFS and LRFS of stage I patients treated with IMRT all reached $100 \%$ during the 5-year follow up, indicating that, with the use of the IMRT, stage I patients had excellent outcomes and low risk to occur tumor-related events regardless of EBV DNA levels. Shortening the follow-up time or extending the interval of routine follow-up exams was recommended to stage I patients.

For stage II patients, the annual survival rates of the endpoints between 1 and 5 years were more than $90 \%$. As shown in Fig 2, the survival curves of the endpoints were all smooth prior to year 5 . When combined the survival curve in Fig 2 and the statistical result, we found that 3-year OS, 3-year PFS, 3-year LRFS and 4-year DMFS could function as surrogate endpoints. These results indicated that local-regional failure and distance failure mainly occurred in years three and four, 3-year LRFS and 4-year DMFS were in accordance with 5-year LRFS and 5-year DMFS, respectively. The interpretation may be that stage II patients had also achieved better therapeutic effects because of the progress of the treatment technology. Through different levels of EBV DNA, we could find a general trend that, when the EBV DNA levels were getting higher, the cut-off year of surrogate endpoints would be pushed earlier, which indicated the higher the EBV DNA, the greater the risk of treatment failure, leading to the poorer prognosis. Furthermore, the survival curve would reach a plateau earlier because of the relatively unsatisfactory outcomes. According to the above results, we might shorten the follow-up time or extend the interval of routine follow-up exams individually for the stage II patients.

Our data failed to reveal that OS and PFS at $<5$-year could be used as surrogate endpoints in stage III patients. Currently, the TNM classification is the most commonly used staging system to reflect the heterogeneity of NPC patients. However, large variations were reported in the clinical outcomes of patients at the same stage receiving similar treatment strategies prompting the thought that the present staging system is still inadequate for predicting the outcome of NPC patients. In addition, for this group of patients, the use of induction chemotherapy and adjuvant chemotherapy was not uniform, which lead to the large heterogeneity of prognosis. Through examination of the different levels of EBV DNA, we could also find a general trend suggesting that, when EBV DNA levels were getting higher, the year of surrogate endpoints would be earlier. The routine follow-up exam for the stage III patients is still necessary.

For stage IVA patients, the prognosis reported by previous studies remained poor [22, 25]. In our study, the rates of LRFS, DMFS, PFS and OS were $>90 \%,>80 \%,>60 \%$ and $>70 \%$ respectively during 5 -year follow up. As shown in Fig 2, the rates of OS continuously decreased from $96.9 \%$ in the 1 st year to $75.4 \%$ in the 5 th yea. The rates of PFS were nearly the same as the rates of OS, continuously decreasing from $91.2 \%$ in the 1 st year to $69.2 \%$ in 5 th year. The rates of LRFS were slowly decreased from $98.4 \%$ in the 1 st year to $90.9 \%$ in the 5 th year and the rates of DMFS were slowly decreased from $82.5 \%$ in the 3rd year to $80.4 \%$ in the 5 th year. We found that the 3-year LRFS and 3-year DMFS could be surrogate endpoints. By examining different levels of EBV DNA, only the cut-off surrogate year of OS could generally meet the trend mentioned above. The results indicated that, first, the prognosis of such patients is related to the heterogeneity of the chemotherapy regimens and patterns. Second, excellent local control had been reached by IMRT $[26,27]$ and the failure pattern shifts from local to distant progressions [28]. Above all, the first-line therapy and the salvage therapy after progression were becoming more effective so that stage IVA patients could have a relatively long survival period even if recurrence or metastasis occurs; thus, the time of the survival curve plateau was delayed. Based on our data, the routine follow-up exam for the stage IVA patients is recommended.

However, there are several limitations to our study. First, some subgroup sample sizes were too small which might make the results of the study underpowered and selection bias might exist. Second, the chemotherapy regimens in this study were heterogeneous, which would add bias to the results. Third, our study was retrospective and carried out at a single center. In the future, we will design a large sample prospective study to further verify the conclusions of this study.

In conclusion, we performed a retrospective research analysis to expand our observations of evidence for shortening the time of therapeutic evaluation, revealing that OS, PFS, LRFS and DMFS endpoints <5-years could be surrogates in different subsets of NPC patients, especially ones in stages I-II. In addition, there seems to be a trend that with the higher levels of EBV DNA, the cut-off year of surrogate endpoints could be earlier in stage II-IVA patients. Our findings confirmed the idea that the 
surrogate endpoints could help clinicians shorten the time of therapeutic evaluation, design individualized follow-up recommendations, save medical resources, and accelerate future clinical trials and the development of effective therapeutic regimens in the research of NPC. However, long-term follow-up is still needed to observe the late adverse effects, and our results still require further confirmation.

\section{Abbreviations}

NPC: nasopharyngeal carcinoma; IMRT: intensity-modulated radiotherapy; EBV DNA: pretreatment plasma Epstein-Barr viral deoxyribonucleic acid; OS: overall survival; PFS: progression-free survival; LRFS: loco-regional recurrence-free survival; DMFS: distant metastasis-free survival; McNemar's test: analysis of variance; SD: standard deviation.

\section{Acknowledgements}

This work was supported by grants from the Hi-Tech Research and Development Program of China (NO. 2006AA02Z4B4), the National Key R \& D Program of Precise Medical Research of China (NO. 2016YFC0904600).

\section{Notes}

Conception and design of the study: SZ, SRL and CC. Acquisition of data: SZ, SRL, SRD, GNW and SL. Analysis and interpretation of the data: SZ, SRL, CC, HC, XY, WWZ, and XHW. Writing and revision of the manuscript: SZ and SRL. All authors read and approved the final manuscript. This is a retrospective study that we collected clinical information from medical records instead of face-to-face investigation, so that consent to participate was waived.

\section{Competing Interests}

The authors have declared that no competing interest exists.

\section{References}

1. Chang ET, Adami H-O. The enigmatic epidemiology of nasopharyngeal carcinoma. Cancer Epidemiology and Prevention Biomarkers. 2006; 15:1765-1777.

2. Ferlay J, Soerjomataram I, Ervik M, et al. GLOBOCAN 2012 v1. 0, cancer incidence and mortality worldwide: IARC CancerBase No. 11. Lyon, France: International agency for research on cancer. 2013; 19.

3. Jemal A, Bray F, Center MM, et al. Global cancer statistics. CA: a cancer journal for clinicians. 2011; 61:69-90.

4. Lee $\mathrm{AW}, \mathrm{Ma} \mathrm{BB}, \mathrm{Ng} \mathrm{WT}$, et al. Management of nasopharyngeal carcinoma: current practice and future perspective. J Clin Oncol. 2015; 33:3356-3364.

5. Lin J-C, Jan J-S, Hsu C-Y, et al. Phase III study of concurrent chemoradiotherapy versus radiotherapy alone for advanced nasopharyngeal carcinoma: positive effect on overall and progression-free survival. J Clin Oncol. 2003; 21:631-637.

6. Sun Y, Li W-F, Chen N-Y, et al. Induction chemotherapy plus concurrent chemoradiotherapy versus concurrent chemoradiotherapy alone in locoregionally advanced nasopharyngeal carcinoma: a phase 3 , multicentre, randomised controlled trial. Lancet Oncol. 2016; 17:1509-1520.

7. Lee N, Xia P, Quivey JM, et al. Intensity-modulated radiotherapy in the treatment of nasopharyngeal carcinoma: an update of the UCSF experience. Int J Radiat Oncol Biol Phys. 2002; 53:12-22.
8. Sun X, Su S, Chen C, et al. Long-term outcomes of intensity-modulated radiotherapy for 868 patients with nasopharyngeal carcinoma: an analysis of survival and treatment toxicities. Radiother Oncol. 2014; 110:398-403.

9. Chen C, Han F, Zhao C, et al. Treatment results and late complications of 556 patients with locally advanced nasopharyngeal carcinoma treated with radiotherapy alone. Br J Radiol. 2009; 82:452-458.

10. Lee AW, Sze W, Au JS, et al. Treatment results for nasopharyngeal carcinoma in the modern era: the Hong Kong experience. Int J Radiat Oncol Biol Phys. 2005; 61:1107-1116.

11. Buyse M, Burzykowski T, Carroll $\mathrm{K}$, et al. Progression-free survival is a surrogate for survival in advanced colorectal cancer. J Clin Oncol. 2007; 25:5218-5224.

12. Burzykowski T, Buyse M, Piccart-Gebhart MJ, et al. Evaluation of tumor response, disease control, progression-free survival, and time to progression as potential surrogate end points in metastatic breast cancer. J Clin Oncol. 2008; 26:1987-1992.

13. Akamatsu $\mathrm{H}$, Mori K, Naito T, et al. Progression-free survival at 2 years is a reliable surrogate marker for the 5-year survival rate in patients with locally advanced non-small cell lung cancer treated with chemoradiotherapy. BMC cancer. 2014; 14:18.

14. Halabi S, Rini B, Escudier B, et al. Progression-free survival as a surrogate endpoint of overall survival in patients with metastatic renal cell carcinoma. Cancer. 2014; 120:52-60.

15. Chen C, Yi W, Gao J, et al. Alternative endpoints to the 5-year overall survival and locoregional control for nasopharyngeal carcinoma: A retrospective analysis of 2,450 patients. Mol Clin Oncol. 2014; 2:385-392.

16. Rotolo F, Pignon JP, Bourhis J, et al. Surrogate End Points for Overall Survival in Loco-Regionally Advanced Nasopharyngeal Carcinoma: An Individual Patient Data Meta-analysis. J Natl Cancer Inst. 2017; 109(4).

17. Leung S-f, Zee B, Ma BB, et al. Plasma Epstein-Barr viral deoxyribonucleic acid quantitation complements tumor-node-metastasis staging prognostication in nasopharyngeal carcinoma. J Clin Oncol. 2006; 24:5414-5418.

18. Lin J-C, Wang W-Y, Chen KY, et al. Quantification of plasma Epstein-Barr virus DNA in patients with advanced nasopharyngeal carcinoma. N Engl J Med. 2004; 350:2461-2470.

19. Tang L, Chen Q, Guo S, et al. The impact of plasma Epstein-Barr virus DNA and fibrinogen on nasopharyngeal carcinoma prognosis: an observational study. Br J Cancer. 2014; 111:1102.

20. Pan JJ, Ng WT, Zong JF, et al. Proposal for the 8th edition of the AJCC/UICC staging system for nasopharyngeal cancer in the era of intensity-modulated radiotherapy. Cancer. 2016; 122:546-558.

21. Amin MB, Edge SB. AJCC cancer staging manual. Springer; 2017.

22. Tang LL, Chen YP, Mao YP, et al. Validation of the 8th Edition of the UICC/AJCC Staging System for Nasopharyngeal Carcinoma From Endemic Areas in the Intensity-Modulated Radiotherapy Era. J Natl Compr Canc Netw. 2017; 15:913-919.

23. Tang LQ, Li CF, Li J, et al. Establishment and Validation of Prognostic Nomograms for Endemic Nasopharyngeal Carcinoma. J Natl Cancer Inst. 2016; 108 .

24. Sun X, Su S, Chen C, et al. Long-term outcomes of intensity-modulated radiotherapy for 868 patients with nasopharyngeal carcinoma: an analysis of survival and treatment toxicities. Radiother Oncol. 2014; 110:398-403.

25. Kang M, Zhou P, Li G, et al. Validation of the 8th edition of the UICC/AJCC staging system for nasopharyngeal carcinoma treated with intensity-modulated radiotherapy. Oncotarget. 2017; 8:70586.

26. Ng WT, Lee MC, Hung WM, et al. Clinical outcomes and patterns of failure after intensity-modulated radiotherapy for nasopharyngeal carcinoma. Int $\mathrm{J}$ Radiat Oncol Biol Phys. 2011; 79:420-428.

27. Yang $B, X u B, Z$ Zeng $Q$, et al. A specialized course of basic skills training for single-port laparoscopic surgery. Surgery. 2011; 149:766-775.

28. Chua ML, Wee JT, Hui EP, et al. Nasopharyngeal carcinoma. Lancet. 2016; 387:1012-1024. 